\title{
Extended Abstract - CTDSI/CTCCSI 2021 - Study and definition of project attributes for selection of testing techniques for concurrent software
}

\author{
Autor: Italo Santos ${ }^{1}$, \\ Orientador: Simone do Rocio Senger de Souza ${ }^{1}$, \\ Co-orientador: Silvana Morita Melo ${ }^{2}$ \\ ${ }^{1}$ Institute of Mathematics and Computer Science \\ University of São Paulo (USP) - Brazil \\ ${ }^{2}$ Federal University of Grande Dourados (UFGD) - Brazil \\ italo.santos@alumni.usp.br, srocio@icmc.usp.br, silvanamelo@ufgd.edu.br
}

\begin{abstract}
Context:] The choice of testing technique to be adopted in a software project persists based on the tester's knowledge and often does not consider all of the testing techniques available in the industry or academia. [Objective:] Our work aims to help testers to select a better testing technique to increase the efficiency of the software test execution process, which in turn influences the development and delivery of a more robust and quality product. [Methodology:] We conduct a systematic mapping study to identify and analyze papers that represent the current state of the literature about testing techniques selection. We surveyed software testing practices carried in Brazil software companies and identified the testing practices to know and have an overview on the latest testing techniques and the selection testing technique process. [Results and Conclusions:] With this study, it is expected to specify project attributes that can be used to improve the existing recommendation system in the SeleCTT tool and propose ways of combining testing techniques, contributing to industry and academia.
\end{abstract}

\section{Context and Motivation}

Software testing is one of the verification, validation, and test (VV\& T) activities that have been used to increase the software quality and developed model [Myers et al. 2011]. Adopting testing techniques in a project relays on the knowledge of the tester and often does not consider all of the testing techniques available in the industry or academia [Vegas and Basili 2005]. Therefore, [Melo et al. 2019] proposed a body of knowledge that brings together, information relevant to the decision making process on which testing technique to apply to a particular software project, a framework was developed to support the characterization and systematic selection of concurrent software testing techniques. Concerning to allow the community to interact with the proposed framework, in the work of [Melo et al. 2019] the SeleCTT ${ }^{1}$ (An infrastructure for Selection of Concurrent Software Testing Techniques) tool was developed and it allows access to the body of knowledge and automates the selection process.

Based on the characteristics of the software project under test and the attributes of the testing techniques presented in the body of knowledge, the SeleCTT tool suggests the

\footnotetext{
${ }^{1}$ http: //www.labes.icmc.usp.br/ selectt/home
} 
best testing technique. However, only one technique is suggested, and the ideal would be to check the possibility of combining more than one technique for improvement of results. Besides, the choice does not take into account information from previous projects. The main objective of this project is to answer the following question: "What project attributes can guide the combined selection of testing techniques for concurrent software?", towards extending and improving the attributes defined by [Melo et al. 2019], and taking into account other characteristics (e.g., information from previous similar projects and combination of testing techniques for improving the results).

\section{Methodology}

1. Systematic mapping study: we identified and analyzed papers that represent the current state-of-the-art of the testing techniques selection [Santos et al. 2019];

2. Brazilian companies survey: a survey on software testing practices conducted in Brazilian software companies provided an overview of the latest testing techniques and the process of testing technique selection;

3. Study of selection attributes: this step was essential for the verification of possible attributes related to concurrent software to use in SeleCTT;

4. Catalog of selection attributes: the study conducted in the previous stage enabled the development of a catalog of selection attributes for assisting testing practitioners in the choice of the most suitable testing techniques [Santos et al. 2020b];

5. Combined selection approach: in the selection process SeleCTT recommends more than one testing technique, considering information related to concurrent bugs and testing level attributes;

6. Evolution of SeleCTT tool: new features were added to SeleCTT towards a combined selection of testing techniques, thus evolving its scope proposed;

7. Evaluation of the combined selection approach: we evaluate the proposed combined selection approach through a case study divided into two scenarios to compare the effectiveness of the recommendations made by SeleCTT and the effectiveness of the recommendations made by the testers [Santos et al. 2020a].

\section{Relationship between our work and Research Challenges in Information Systems}

This masters work involves testing activity that is no longer considered an activity that starts only after the coding phase has been completed and is limited to the detection of failures. It has been widely used in the industry for quality assurance, since, by directly scrutinizing the software in execution, it provides realistic feedback of the software behavior, and remains the essential complement to other analysis techniques. In this context, and with the growing demand for information systems (IS) as agents of economic growth and social transformations in Brazil and in the world in the last decades [Araujo et al. 2017]. Testing activity is an important ally to support the development of IS that arise to cover the needs of companies and today's society.

Software testing practitioners must make decisions on techniques to be used in a specific situation and estimate the time and efforts necessary for their application. However, the literature reports no clear guidelines for such a task [Vos et al. 2012]. The correct selection of testing techniques influences the quality of both process and product. Several testing techniques are available, and each of them has different and often complementary features that test several aspects of the software. 


\section{Conclusions}

This master work arises from the need to help testers to improve the way they perform the task of testing techniques selection in their work environment. During the masters, we tried to approach this problem from different perspectives by doing a systematic mapping study to gather papers that represent the current state-of-the-art of testing techniques selection. We also surveyed testers in the industry to understand their main practices and challenges faced. We evaluated the selection attributes used in previous work and create a catalog that could be used as an artifact to help testers based the selection process of testing techniques. Moreover, we developed an approach to support the combined selection of software testing techniques for concurrent programs. Our study described SeleCTT-v2 which was developed to help testers to select a combination of testing techniques, recommending to testers which will be the most suitable combination taking into account the software project characteristics provided by the tester.

\section{Acknowledgements}

The authors acknowledge FAPESP (São Paulo Research Foundation), for the financial support under process number 2018/10183-9.

\section{Referências}

Araujo, R. M., Maciel, R. S., and Boscarioli, C. (2017). I grandsi-br-grandes desafios de pesquisa em sistemas de informação no brasil 2016 a 2026. relatório técnico, 67pg.

Melo, S. M., Moura, F. M., Souza, P. S. L., and Souza, S. R. S. (2019). SeleCTT: An infrastructure for selection of concurrent software testing techniques. In Proceedings of the IV Brazilian Symposium on Systematic and Automated Software Testing, SAST 2019, page 62-71, New York, NY, USA. Association for Computing Machinery.

Myers, G. J., Sandler, C., and Badgett, T. (2011). The art of software testing. John Wiley $\&$ Sons.

Santos, I., Furlanetti, A. B., Melo, S. M., Souza, P. S. L., Delamaro, M. E., and Souza, S. R. (2020a). Contributions to improve the combined selection of concurrent software testing techniques. In Proceedings of the 5th Brazilian Symposium on Systematic and Automated Software Testing, pages 69-78.

Santos, I., Melo, S. M., Souza, P. S. L., and Souza, S. R. (2020b). Towards a unified cata$\log$ of attributes to guide industry in software testing technique selection. In 2020 IEEE International Conference on Software Testing, Verification and Validation Workshops (ICSTW), pages 398-407. IEEE.

Santos, I., Melo, S. M., Souza, P. S. L., and Souza, S. R. S. (2019). Testing techniques selection: A systematic mapping study. In Proceedings of the XXXIII Brazilian Symposium on Software Engineering, pages 347-356. ACM.

Vegas, S. and Basili, V. (2005). A characterisation schema for software testing techniques. Empirical Software Engineering, 10(4):437-466.

Vos, T. E., Marin, B., Escalona, M. J., and Marchetto, A. (2012). A methodological framework for evaluating software testing techniques and tools. In 2012 12th international conference on quality software, pages 230-239. IEEE. 